RAIRO-Inf. Theor. Appl. 40 (2006) 511-518

DOI: 10.1051/ita:2006031

\title{
A LITTLE MORE ABOUT MORPHIC STURMIAN WORDS
}

\author{
ISABELLE FAGNOT ${ }^{1}$
}

\begin{abstract}
Among Sturmian words, some of them are morphic, i.e. fixed point of a non-identical morphism on words. Berstel and Séébold (1993) have shown that if a characteristic Sturmian word is morphic, then it can be extended by the left with one or two letters in such a way that it remains morphic and Sturmian. Yasutomi (1997) has proved that these were the sole possible additions and that, if we cut the first letters of such a word, it didn't remain morphic. In this paper, we give an elementary and combinatorial proof of this result.
\end{abstract}

Mathematics Subject Classification. 68R15, 68Q45.

\section{INTRODUCTION}

Sturmian words have been yet widely studied, they can be viewed as approximation of half-lines whose slopes are irrational numbers between 0 and 1 . A combinatorial approach consists in saying that they are the only infinite words over a binary alphabet to have exactly $n+1$ factors of length $n$. Given a half-line $y=\alpha x+\rho, 0<\alpha<1$, we denote by $s_{\alpha, \rho, \downarrow}\left(\right.$ resp. $s_{\alpha, \rho, \uparrow}$ ) the lower approximation (resp. the upper approximation) of this half-line.

Among Sturmian words, some of them are also morphic, i.e. fixed point of a non-identical morphism on words. The most famous one is undoubtedly the so-called Fibonacci word whose generating morphism is $\varphi: 0 \mapsto 01,1 \mapsto 0$. This word corresponds to $s_{\frac{1}{\tau^{2}}, \frac{1}{\tau^{2}}, \downarrow}$ with $\tau=\frac{1+\sqrt{5}}{2}$.

If we erase the first $p$ letters of a Sturmian word $s_{\alpha, \rho, d}$, we get another Sturmian word $s_{\alpha, \rho+p \alpha, d}$. Quite symetrically, if we add $p$ well chosen letters before a

Keywords and phrases. Sturmian words, infinite words, iterated morphisms, combinatorics of words.

${ }^{1}$ Institut Gaspard Monge, Cité Descartes, 5, boulevard Descartes, Champs-sur-Marne, 77454 Marne-la-vallée cedex 2, France, fagnot@univ-mlv.fr

(c) EDP Sciences 2006 
Sturmian word $s_{\alpha, \rho, d}$, we get another Sturmian words of the form $s_{\alpha, \rho-p \alpha, d^{\prime}}$. But if such a Sturmian word is morphic will we get a morphic word?

In one hand, Berstel and Séébold [2] proved that a restricted category of Sturmian words (the characteristic ones) remains morphic if we add one or two letters.

On the other hand, Parvaix [8] gives a necessary condition on the slope $(\alpha)$ of the Sturmian word so that it is morphic (see also Crisp, Moran, Pollington and Shiue [5] and Allauzeen [1]). Moreover, Yasutomi [9] has given an algebraic characterization of Sturmian words that are also morphic, this characterization in on the slope $(\alpha)$ and the intercept $(\rho)$. A corollary of this characterization is that, given a morphic characteristic word, we can't add more than two letters, if we want it to remain morphic and Sturmian, and we cannot remove any of the beginning sequences of letters from it. Berthé, Ei, Ito and Rao [4] give another proof of Yasutomi's theorem and corollary. Both latter articles mainly use number theory.

The aim of this paper is to give a more elementary and combinatorial proof of the Yasutomi's corollary.

Note that, this latter result has been recently extended to episturmian morphisms by Justin and Pirillo [6].

Section 2 will be devoted to definitions of Sturmian words. In Section 3, previous results about Sturmian morphic words will be cited as well as our result, the proof of which will be done in the last section.

\section{STURMiAn WORDS}

There are many definitions and properties related to Sturmian words. Here, we only recall those we are going to use. For more information about Sturmian words, the reader is referred to the survey of Berstel and Séébold [3].

Let $A$ be the finite alphabet $\{0,1\}$. The set of finite words is denoted by $A^{*}$ and the set of infinite words by $A^{\omega}$. The empty word is denoted by $\varepsilon$.

Given two real numbers $\alpha$ and $\rho, 0<\alpha<1$, we denote $s_{\alpha, \rho, \uparrow}=a_{0} a_{1} \ldots a_{n} \ldots$ and $s_{\alpha, \rho, \downarrow}=b_{0} b_{1} \ldots b_{n} \ldots$ words over the alphabet $\{0,1\}$ with

$$
\begin{aligned}
& a_{n}=\lfloor(n+1) \alpha+\rho\rfloor-\lfloor n \alpha+\rho\rfloor \\
& b_{n}=\lceil(n+1) \alpha+\rho\rceil-\lceil n \alpha+\rho\rceil .
\end{aligned}
$$

These words are said to be Sturmian if $\alpha$ is irrational. Then $\alpha$ is called the slope and $\rho$ the intercept. Note that $s_{\alpha, \rho, \downarrow}=s_{\alpha, \rho, \uparrow}$ unless there exists an integer $n \geq 0$ such that $n \alpha+\rho \in \mathbb{N}$.

A geometric approach of Sturmian word is the following: we draw the half-line of equation $y=\alpha x+\rho, y \geq 0$ and try to approximate it by horizontal $\_-$and diagonal unit segments this by $\__{0}$ is 0 and $\mathcal{L}^{\rho}$ is 1 , the lower approximation gives $s_{\alpha, \rho, \downarrow}$ and the upper one gives $s_{\alpha, \rho, \uparrow}^{\prime}$ (see Fig. 1). 


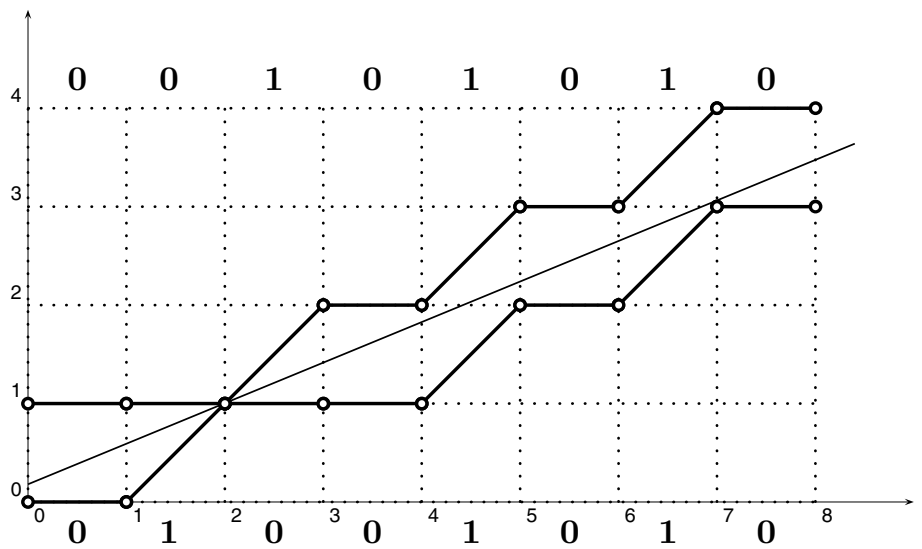

FiguRE 1. Geometric representation of Sturmian words.

The characteristic Sturmian word of slope $\alpha$ is the word $c_{\alpha}=s_{\alpha, \alpha, \downarrow}=s_{\alpha, \alpha, \uparrow}$. It corresponds to the line $y=\alpha x+\alpha$.

Remark 1. Note that $s_{\alpha, \rho, d}=s_{\alpha, \rho+n, d}$ for any $n \in \mathbb{N}$.

Remark 2. Note also that if we erase the first $p$ letters of the Sturmian word $s_{\alpha, \rho, d}$, we get $s_{\alpha, \rho+p \alpha, d}$. (It is just a horizontal shift of the origin of the halfline.) Symetrically, if we add $p$ (cautiously chosen) letters at the Sturmian word $s_{\alpha, \rho+p \alpha, d}$, we get $s_{\alpha, \rho+p \alpha, d^{\prime}}$. (We may have one or two possibilities.)

\section{STURMIAN MORPHISMS}

An (endo)morphism over an alphabet $B$ is a function $f: B^{*} \rightarrow B^{*}$ such that for any couple of words $u, v \in B^{*}$, we have $f(u v)=f(u) f(v)$. If the morphism $f$ is non-erasing, (i.e. if there exists no letter $a$ such that $f(a)=\varepsilon$ ) and if there exists a letter $a$ such that $f(a)=a v$, with $v \neq \varepsilon$ then there exists one infinite word $\mathbf{x}$ such that $\mathbf{x}=\lim _{n \rightarrow \infty} f(a)$. This word $\mathbf{x}$ is said to be morphic.

A morphism $f: A^{*} \rightarrow A^{*}$ is said to be Sturmian if for every Sturmian word $\mathbf{x}$, the word $f(\mathbf{x})$ is also Sturmian.

Let $E, \varphi$ and $\tilde{\varphi}$ be the morphisms

$$
E: \begin{array}{lll}
0 & \mapsto & 1 \\
1 & \mapsto & 0
\end{array} \quad \varphi: \begin{array}{lllll}
0 & \mapsto & 01 \\
1 & \mapsto & 0
\end{array} \quad \tilde{\varphi}: \begin{array}{lll}
0 & \mapsto 10 \\
1 & \mapsto 0
\end{array}
$$

Mignosi and Séébold characterize the Sturmian morphisms as following.

Theorem 3 [7]. A morphism is Sturmian if and only if it is a composition of the three morphisms $E, \varphi$ and $\tilde{\varphi}$.

As in Parvaix [8], we will denote $G=\varphi E$ and $D=\tilde{\varphi} E$. Noticing that $E E=I d$, it is easy to see that we have again that 
Remark 4 [8]. A morphism is Sturmian if and only if it is a composition of the three morphisms $E, G$ and $D$.

What is more interesting is the exact description of the action of those morphisms $E, G$ and $D$, that Parvaix gives.

Proposition 5 Parvaix [8]. Let $\alpha \in] 0,1[$ be an irrational number. If, moreover, $\rho \in[0,1[$, we have

$$
\begin{aligned}
E\left(s_{\alpha, \rho, \downarrow}\right) & =s_{1-\alpha, 1-\rho, \uparrow} \\
G\left(s_{\alpha, \rho}, \downarrow\right) & =s_{\frac{\alpha}{\alpha+1}, \frac{\rho}{\alpha+1}, \downarrow} \\
D\left(s_{\alpha, \rho}, \downarrow\right) & =s_{\frac{\alpha}{\alpha+1}, \frac{\rho+\alpha}{\alpha+1}, \downarrow}
\end{aligned}
$$

and symmetrically, if $\rho \in] 0,1]$, we have

$$
\begin{aligned}
& E\left(s_{\alpha, \rho, \uparrow}\right)=s_{1-\alpha, 1-\rho, \downarrow} \\
& G\left(s_{\alpha, \rho, \uparrow}\right)=s_{\frac{\alpha}{\alpha+1}, \frac{\rho}{\alpha+1}, \uparrow} \\
& D\left(s_{\alpha, \rho, \uparrow}\right)=s_{\frac{\alpha}{\alpha+1}, \frac{\rho+\alpha}{\alpha+1}, \uparrow}
\end{aligned}
$$

Moreover, in the same article, he gives a necessary condition for a Sturmian word to be morphic.

Theorem 6 Parvaix [8]. Let $\mathbf{x}$ be a Sturmian word of slope $\alpha$. If there exists a morphism $f$ such that $f(\mathbf{x})=\mathbf{x}$, then $\alpha$ is a Sturm number, that is

- either $\alpha=\left[0,1+k_{n}, \overline{k_{n-1}, \ldots k_{2}, k_{1}+k_{n}}\right]$ with $n \geq 2,\left(k_{1}, k_{n}\right) \in \mathbb{N}^{2} \backslash$ $\{(0,0)\}$ and $k_{j} \in \mathbb{N} \backslash 0$, for $2 \leq j \leq n-1$,

- either $\alpha=\left[0,1, k_{n}, \overline{k_{n-1}, \ldots k_{2}, k_{1}+k_{n}}\right]$ with $n \geq 2$ and $k_{j} \in \mathbb{N} \backslash\{0\}$, for $2 \leq j \leq n$.

Reversely, he proves that

Theorem 7 Parvaix [8]. If $\alpha$ is a Sturm number, then $c_{\alpha}=s_{\alpha, \alpha, \downarrow}=s_{\alpha, \alpha, \uparrow}, s_{\alpha, 0, \downarrow}$, $s_{\alpha, 0, \uparrow}, s_{\alpha,-\alpha, \downarrow}$ and $s_{\alpha,-\alpha, \downarrow}$ are morphic.

He also gives a description of the generating morphisms based on the continued expansion of $\alpha$.

Part of this result was already known in a different formulation by Berstel and Séébold

Theorem 8 Berstel, Séébold [2]. If the Sturmian characteristic word $c_{\alpha}$ is morphic, then it is also the case for $0 c_{\alpha}=s_{\alpha, 0, \downarrow}, 1 c_{\alpha}=s_{\alpha, 0, \uparrow}, 01 c_{\alpha}=s_{\alpha,-\alpha, \downarrow}$ and $10 c_{\alpha}=s_{\alpha,-\alpha, \uparrow}$.

The morphisms generating these words are here built on the morphism generating $c_{\alpha}$.

Yasutomi proved that these are the sole completions of characteristic Sturmian words to be morphic and Sturmian. And moreover that deleting the first letters of a characteristic word does not produce a morphic word either. That is 
Theorem 9 Yasutomi [9], Berthé, Ei, Ito and Rao [4]. Given an irrational $\alpha$, $0<\alpha<1$ such that the characteristic word $c_{\alpha}$ is morphic, the only Sturmian words of the form $s_{\alpha, p \alpha, \downarrow}$ or $s_{\alpha, p \alpha, \uparrow}$ with $p \in \mathbb{Z}$ which are morphic too are the following

$$
\begin{aligned}
& c_{\alpha}=s_{\alpha, \alpha, \downarrow}=s_{\alpha, \alpha, \uparrow} \\
& s_{\alpha, 0, \downarrow}=0 c_{\alpha} \text { and } s_{\alpha, 0, \uparrow}=1 c_{\alpha} \\
& s_{\alpha,-\alpha, \downarrow}=01 c_{\alpha} \text { and } s_{\alpha,-\alpha, \uparrow}=10 c_{\alpha} .
\end{aligned}
$$

Remark 10. By Theorems 6 and 7, the condition " $c_{\alpha}$ is morphic" is equivalent to " $\alpha$ is a Sturm number".

Remark 11. If $p$ is a non-positive integer, $s_{\alpha, p \alpha, \downarrow}$ and $s_{\alpha, p \alpha, \uparrow}$ are the unique Sturmian words obtained by adding $-p+1$ letters before the beginning of $c_{\alpha}$.

If $p$ is positive, $s_{\alpha, p \alpha, \downarrow}=s_{\alpha, p \alpha, \uparrow}$ is obtained by removing $p-1$ letters in the beginning of $c_{\alpha}$.

The next section is devoted to a new proof of the necessary part of the latter theorem, that is that there are no other solutions that those found by Berstel and Séébold [2].

\section{A NeW PRoof of Theorem 9(PART OF)}

\subsection{Notations AND BASIC FORMUlae}

Let $d \in\{\downarrow, \uparrow\}$ we will denote $\bar{d}$ the element of $\{\downarrow, \uparrow\}$ such that $\bar{d} \neq d$.

To prove this theorem, we must consider the words $s_{\alpha, p \alpha, d}, 0<\alpha<1, p \in \mathbb{Z}$ and $d \in\{\downarrow, \uparrow\}$.

Note that, if $q=-\lfloor p \alpha\rfloor$, we have $0<\rho=p \alpha+q<1$ and $s_{\alpha, \rho, d}=s_{\alpha, p \alpha, d}$. In the sequel, in order to be able to use Proposition 5 , we will only consider words of the form $s_{\alpha, p \alpha+q, d}$ with $\alpha$ irrational, $0<\alpha<1$ and $p, q \in \mathbb{Z}$ such that $\left.p \alpha+q \in\right] 0,1[$ and $d \in\{\downarrow, \uparrow\}$.

Let $\mathcal{S}$ be the set of these words:

$$
\mathcal{S}=\left\{s_{\alpha, p \alpha+q, d} \mid \alpha \text { irrational, } 0<\alpha<1, p, q \in \mathbb{Z}, p \alpha+q \in\right] 0,1[, d \in\{\downarrow, \uparrow\}\} .
$$

Note that $s_{\alpha, p \alpha+q, d}=s_{\alpha^{\prime}, p^{\prime} \alpha^{\prime}+q^{\prime}, d^{\prime}}$ if and only if $\alpha=\alpha^{\prime}, p=p^{\prime}, q=q^{\prime}, d=d^{\prime}$ or $\alpha=\alpha^{\prime}, p=p^{\prime}>0, q=q^{\prime}, d^{\prime}=\bar{d}$. We will study the evolution of these words under the action of the morphisms $E, G$ and $D$.

The reader can easily check that Proposition 5 can leads to

$$
\begin{aligned}
E\left(s_{\alpha, p \alpha+q, d}\right) & =s_{1-\alpha, p(1-\alpha)+1-p-q, \bar{d}} \\
G\left(s_{\alpha, p \alpha+q, d}\right) & =s_{\frac{\alpha}{\alpha+1},(p-q) \frac{\alpha}{\alpha+1}+q, d} \\
D\left(s_{\alpha, p \alpha+q, d}\right) & =s_{\frac{\alpha}{\alpha+1},(p-q+1) \frac{\alpha}{\alpha+1}+q, d}
\end{aligned}
$$

with $\varepsilon \in\{0,1\}$. 
It will be convenient to define functions $P, Q$ and $A$ by

$$
\begin{aligned}
& P\left(s_{\alpha, p \alpha+q, d}\right)=p \\
& Q\left(s_{\alpha, p \alpha+q, d}\right)=q \\
& A\left(s_{\alpha, p \alpha+q, d}\right)=\alpha .
\end{aligned}
$$

Thus we have, for any Sturmian word $\mathbf{x} \in \mathcal{S}$ and $n \in \mathbb{N}$

$$
\begin{aligned}
P(E(\mathbf{x})) & =P(\mathbf{x}) \\
P(G(\mathbf{x})) & =P(\mathbf{x})-Q(\mathbf{x}) \\
P(D(\mathbf{x})) & =P(\mathbf{x})-Q(\mathbf{x})+1 \\
Q(E(\mathbf{x})) & =1-P(\mathbf{x})-Q(\mathbf{x}) \\
Q(G(\mathbf{x})) & =Q(\mathbf{x}) \\
Q(D(\mathbf{x})) & =Q(\mathbf{x}) \\
A(E(\mathbf{x})) & =1-A(\mathbf{x}) \\
A\left(G^{n}(\mathbf{x})\right) & =\frac{A(\mathbf{x})}{n A(\mathbf{x})+1} \\
A\left(D^{n}(\mathbf{x})\right) & =\frac{A(\mathbf{x})}{n A(\mathbf{x})+1} .
\end{aligned}
$$

\subsection{Proof}

The proof is by contradiction. Let $\mathbf{x}=s_{\alpha, p \alpha+q, d} \in \mathcal{S}$ with $p \notin\{-1,0,1\}$. We assume there exists a non-trivial Sturmian morphism $f$ such that $f(\mathbf{x})=\mathbf{x}$ and we will prove that it is not possible.

By Remark 4, we have $f \in\{E, G, D\}^{*}$. Let $f=g_{n} g_{n-1} \cdots g_{1}$ with $g_{i} \in$ $\{E, G, D\}$ for $i, 1 \leq i \leq n$ a decomposition of $f$. As $E E=I d$, we can suppose that there is no $i, 1 \leq i \leq n-1$ such that $g_{i}=E$ and $g_{i+1}=E$. Let the Sturmian words $\mathbf{x}_{i} \in \mathcal{S}$ be $\mathbf{x}_{i}=g_{i} g_{i-1} \cdots g_{1}(\mathbf{x})$, for $i, 0 \leq i \leq n$. Of course, we have $\mathbf{x}_{n}=f(\mathbf{x})=\mathbf{x}=\mathbf{x}_{0}$ and $\mathbf{x}_{i}=g_{i}\left(\mathbf{x}_{i-1}\right)$.

We will consider two cases : $P(\mathbf{x})=p \geq 2$ and $P(\mathbf{x})=p \leq-2$.

$\mathbf{P}(\mathbf{x}) \geq \mathbf{2}$ : Firstly, let us prove that $f \in\{E, G\}^{*}$ and that $g_{i}=G$ implies $Q\left(\mathbf{x}_{i}\right)=0$.

We can check by induction that $i \rightarrow P\left(\mathbf{x}_{i}\right)$ is increasing and superior to 2. Indeed, the base case is obvious as we suppose $P(\mathbf{x})=P\left(\mathbf{x}_{0}\right) \geq$ 2. Then, if we suppose $P\left(\mathbf{x}_{i}\right) \geq 2$, then $Q\left(\mathbf{x}_{i}\right)=-\left\lfloor P\left(\mathbf{x}_{i}\right) A\left(\mathbf{x}_{i}\right)\right\rfloor \leq 0$, thus $P\left(E\left(\mathbf{x}_{i}\right)\right)=P\left(\mathbf{x}_{i}\right) \geq P\left(\mathbf{x}_{i}\right), P\left(G\left(\mathbf{x}_{i}\right)\right)=P\left(\mathbf{x}_{i}\right)-Q\left(x_{i}\right) \geq P\left(\mathbf{x}_{i}\right)$ and $P\left(D\left(\mathbf{x}_{i}\right)\right)=P\left(\mathbf{x}_{i}\right)-Q\left(x_{i}\right)+1>P\left(\mathbf{x}_{i}\right)$. Then, whatever $g_{i+1}$ is, $P\left(\mathbf{x}_{i+1}\right)=P\left(g_{i+1}\left(x_{i}\right)\right) \geq P\left(\mathbf{x}_{i}\right)$. 
Thus $P\left(\mathbf{x}_{i}\right)$ is increasing, but, as we want $\mathbf{x}_{0}=\mathbf{x}_{n}$, we must have $P\left(\mathbf{x}_{0}\right)=P\left(\mathbf{x}_{1}\right)=\cdots P\left(\mathbf{x}_{n}\right)$, and subsequently we cannot have $g_{i}=D$ for any $i$.So we know that $f \in\{E, G\}^{*}$.

Moreover, if, for one $i$, we have $g_{i}=G$, thus as $P\left(x_{i}\right)=P\left(G\left(\mathbf{x}_{i-1}\right)\right)=$ $P\left(\mathbf{x}_{i-1}\right)-Q\left(\mathbf{x}_{i-1}\right)=P\left(\mathbf{x}_{i-1}\right)$ we must also have that $Q\left(\mathbf{x}_{i-1}\right)=0$ and then $Q\left(\mathbf{x}_{i}\right)=0$.

We are again obliged to distinguish different cases, each one leading to a contradiction.

1. Suppose the decomposition of $f$ contains the sequence $G E G$, that is, assume that $g_{p}=G, g_{p+1}=E$ and $g_{p+2}=G$ for some $p, 1 \leq$ $p \leq n-2$. As $g_{p}=G$, we have $Q\left(\mathbf{x}_{p-1}\right)=0$. Then we have also $Q\left(\mathbf{x}_{p}\right)=Q\left(G\left(\mathbf{x}_{p-1}\right)\right)=0$. Thus $Q\left(\mathbf{x}_{p+1}\right)=Q\left(E\left(\mathbf{x}_{p}\right)\right)=1-P\left(\mathbf{x}_{p}\right)-$ $Q\left(\mathbf{x}_{p}\right)=1-P(\mathbf{x})$. And since $g_{p+2}=G, Q\left(\mathbf{x}_{p+1}\right)=0$ and then $P(\mathbf{x})=1$ which is a contradiction with the assumption.

At this point, we can say that $f \in E G^{+} E \cup E G^{+} \cup G^{+} E \cup G^{+}$.

2. Suppose $f=E G^{n-1}, n \geq 2$ i.e. $g_{1}=G, \ldots, g_{n-1}=G, g_{n}=E$. As $g_{1}=G$ we have $Q(\mathbf{x})=0$ and therefore $Q\left(\mathbf{x}_{n-1}\right)=Q\left(G^{n-1}(\mathbf{x})\right)=0$. Therefore $Q\left(\mathbf{x}_{n}\right)=Q\left(E\left(\mathbf{x}_{n-1}\right)\right)=1-P\left(\mathbf{x}_{n-1}\right)-Q\left(\mathbf{x}_{n-1}\right)=1-P(\mathbf{x})$. As $\mathbf{x}_{n}=\mathbf{x}$ we have $Q(\mathbf{x})=0=1-P(\mathbf{x})$ and therefore $P(\mathbf{x})=1$. It is again contradictory.

3. Suppose $f=G^{n-1} E, n \geq 2$. Let $f^{\prime}=E G^{n-1}=E f E$ and $\mathbf{t}=E(\mathbf{x})$. Then we can check that $f^{\prime}(\mathbf{t})=\mathbf{t}$. Thus $P(\mathbf{t})=1$ (cf. Case 2) and then $P(\mathbf{x})=P(E(\mathbf{t}))=P(\mathbf{t})=1$ which is a contradiction with the assumption.

4. Suppose $f=G^{n}$ i.e. $g_{1}=G, \ldots, g_{n}=G$. Then, we can check that $A(f(\mathbf{x}))=\frac{A(\mathbf{x})}{n A(\mathbf{x})+1}$. As $A(\mathbf{x}) \neq 0$, this implies $n=0$, i.e. $f=I d$. A contradiction.

5. Suppose $f=E G^{n-2} E, n \geq 3$, Let $f^{\prime}=G^{n-2}=E f E$ and $\mathbf{t}=E(\mathbf{x})$. Then we can check that $f^{\prime}(\mathbf{t})=\mathbf{t}$. Thus $n-2=0$ (cf. Case 4) and then $f^{\prime}=I d$ and $f=I d$ which is a contradiction.

6. Suppose $f=E$. Then $A(E(\mathbf{x}))=1-A(\mathbf{x})=A(\mathbf{x})$ and therefore $A(\mathbf{x})=\frac{1}{2}$, which is not an irrational number.

So, if $P(\mathbf{x}) \geq 2$ there is no non-trivial morphism such that $f(\mathbf{x})=\mathbf{x}$.

$\mathbf{P}(\mathbf{x}) \leq-\mathbf{2}$ : The proof of this case is quite similar to the previous one. The author apologizes for not having managed to avoid it.

Here we have, $Q(\mathbf{x})=-\lfloor P(\mathbf{x}) A(\mathbf{x})\rfloor \geq-P(\mathbf{x}) A(\mathbf{x}) \geq-1$. One can check by induction that the sequence $P(\mathbf{x}), P\left(\mathbf{x}_{1}\right), \ldots P\left(\mathbf{x}_{n}\right)$ is decreasing and that $Q\left(\mathbf{x}_{i}\right) \geq 1$. Since $P\left(\mathbf{x}_{n}\right)=P(\mathbf{x})$, this implies $P(\mathbf{x})=$ $P\left(\mathbf{x}_{1}\right)=\cdots P\left(\mathbf{x}_{n}\right)$ and then $g_{i} \in\{E, D\}$ and in the case where $g_{i}=D$, as $P\left(D\left(\mathbf{x}_{i-1}\right)\right)=P\left(\mathbf{x}_{i-1}\right)-Q\left(\mathbf{x}_{i-1}\right)+1$, we must have also $Q\left(\mathbf{x}_{i-1}\right)=1$.

We now consider the following different cases.

1. Suppose the decomposition of $f$ contains the sequence $D E D$, that is, assume that $g_{p}=D, g_{p+1}=E$ and $g_{p+2}=D$ for some $p, 1 \leq p \leq$ $n-2$. As $g_{p}=D$, we have $Q\left(\mathbf{x}_{p-1}\right)=1$. Then we have also $Q\left(\mathbf{x}_{p}\right)=$ $Q\left(D\left(\mathbf{x}_{p-1}\right)\right)=1$. Thus $Q\left(\mathbf{x}_{p+1}\right)=Q\left(E\left(\mathbf{x}_{p}\right)\right)=1-P\left(\mathbf{x}_{p}\right)-Q\left(\mathbf{x}_{p}\right)=$ 
$-P(\mathbf{x})$. And since $g_{p+2}=D, Q\left(\mathbf{x}_{p+1}\right)=1$ and then $P(\mathbf{x})=-1$ which is a contradiction with the assumption.

2. Suppose $f=E D^{n-1}, n \geq 2$ i.e. $g_{1}=D, \ldots, g_{n-1}=D, g_{n}=E$. As $g_{1}=D$ we have $Q(\mathbf{x})=1$ and therefore $Q\left(\mathbf{x}_{n-1}\right)=Q\left(D^{n-1}(\mathbf{x})\right)=1$. Therefore $Q\left(\mathbf{x}_{n}\right)=Q\left(E\left(\mathbf{x}_{n-1}\right)\right)=1-P\left(\mathbf{x}_{n-1}\right)-Q\left(\mathbf{x}_{n-1}\right)=-P(\mathbf{x})$. As $\mathbf{x}_{n}=\mathbf{x}$ we have $Q(\mathbf{x})=1=-P(\mathbf{x})=Q\left(\mathbf{x}_{n}\right)$ and therefore $P(\mathbf{x})=$ -1 . It is again contradictory.

3. Suppose $f=D^{n-1} E$. Let $f^{\prime}=E D^{n-1}=E f E$ and $\mathbf{t}=E(\mathbf{x})$. Then we can check that $f^{\prime}(\mathbf{t})=\mathbf{t}$. Thus $P(\mathbf{t})=-1$ (cf. Case 2) and then $P(\mathbf{x})=P(E(\mathbf{t}))=P(\mathbf{t})=1$ which is a contradiction with the assumption.

4. Assume $f=D^{n}, f=E D^{n-2} E$ or $f=E$. For these three cases the proof is exactly the same as in the case where $P(\mathbf{x}) \geq 2$ and $f=G^{n}$ or $f=E G^{n-2} E$, because it only uses the function $A$ whose evolution under the action of $G$ and $D$ is the same.

So, if $P(\mathbf{x}) \geq 2$ there is no non-trivial morphism such that $f(\mathbf{x})=\mathbf{x}$.

Acknowledgements. First, I wish to thank Jean Bertel for giving me the subject of this article. I also thank both referees for their corrections and suggestions and particularly for having helped me to simplify the proof.

\section{REFERENCES}

[1] C. Allauzen, Une caractérisation simple des nombres de Sturm. J. Théor. Nombres Bordeaux 10 (1998) 237-241.

[2] J. Berstel and P. Séébold, A remark on morphic sturmian words. Theor. Inform. Appl. 28 (1994) 255-263.

[3] J. Berstel and P. Séébold, Algebraic combinatorics on Words, chapter Sturmian words. Cambridge University Press (2002).

[4] V. Berthé, H. Ei, S. Ito and H. Rao, Invertible susbtitutions and Sturmian words: an application of Rauzy fractals. Preprint.

[5] D. Crisp, W. Moran, A. Pollington and P. Shiue, Substitution invariant cutting sequences. J. Théor. Nombres Bordeaux 5 (1993) 123-137.

[6] J. Justin and G. Pirillo, Episturmian words: Shifts, morphisms and numeration systems. Inter. J. Found. Comput. Sci. 15 (2004) 329-348.

[7] F. Mignosi and P. Séébold, Morphismes sturmiens et règles de Rauzy. J. Théor. Nombres Bordeaux 5 (1993) 221-233.

[8] B. Parvaix, Propriétés d'invariance des mots sturmiens. J. Théor. Nombres Bordeaux 9 (1997) 351-369.

[9] Shin-Ichi Yasutomi, On sturmian sequences which are invariant under some substitutions, in Number theory and its applications. Proceedings of the conference held at the RIMS, Kyoto, Japan, November 10-14, 1997, edited by Kanemitsu, Shigeru et al. Kluwer Acad. Publ. Dordrecht (1999) 347-373.

Communicated by Jean Berstel.

Received 15 April 2005. Accepted 19 September 2005. 